\title{
Model Free Adaptive Control of Switched Reluctance Motor for Electric Vehicle
}

\author{
Xifeng $\mathbf{M i}^{{ }^{1}}$, Yuanyuan Fan $^{1}$ \\ ${ }^{1}$ Jiaozuo Normal College, Jiaozuo, 454000, China \\ *Corresponding Author.
}

\begin{abstract}
:
In this paper, the model free adaptive control method of switched reluctance motor for electric vehicle is studied. Based on the torque distribution control of SRM, a SRM control strategy based on torque current hybrid model based on RBF neural network is proposed in this paper. Based on the deviation between the dynamic average value and instantaneous value of SRM output torque, the online learning of RBF neural network is realized. At the same time, this paper constructs a torque current hybrid model, obtains the current variation law of SRM under low torque ripple operation, and reduces the torque ripple of SRM. The SRM torque distribution control is realized on the SRM experimental platform. Compared with the voltage chopper control method, the experimental results show that the torque ripple of SRM can be reduced by adopting the torque distribution control strategy.
\end{abstract}

Keywords: Forest Road, Electric Vehicle, Switched Reluctance Motor, Adaptive Control.

\section{INTRODUCTION}

In order to achieve the goal of "energy conservation, emission reduction and low-carbon life", our government attaches great importance to the development of electric vehicles. In 2011, China's "two sessions" clearly pointed out in the outline of the 12th Five year plan that pure electric vehicles are the key development objects in the new energy vehicle industry [1-2]. The government has issued a series of preferential policies and provided funds to support the new development of China's pure electric vehicle industry. Pure electric vehicle is a vehicle that uses the electric energy stored in the battery as the driving energy [3]. Zero emission and no pollution are the main development direction of electric vehicle. As the power source of pure electric vehicle, the driving performance of driving motor determines the running state of the vehicle. Therefore, it is important for automotive researchers to choose which motor as the power source. At present, the drive motors used in pure electric vehicles mainly include DC motor, asynchronous motor, permanent magnet brushless DC motor, SRM, etc. At present, 
Article History: Received: 28 October 2021 Revised: 05 December 2021 Accepted: 10 January 2022 Publication: 28 February 2022

SRM drive system has been successfully applied to large vehicles [4-5]. Ge and Sundstrand have developed a test prototype of HVDC SRM starting / power generation system applied to aircraft, which has been successfully applied to the new American fighter F-35 [6]. For large vehicles such as buses and aircraft, the torque ripple of SRM has little impact on its operation performance. However, the pure electric vehicle industry will mainly face the private vehicle field in the future, with higher requirements for vehicle safety and ride comfort. The torque ripple generated during SRM operation hinders its popularization and application in the private pure electric vehicle field [7-8]. Therefore, the research on the method of restraining SRM torque ripple has extremely important theoretical and engineering significance.

\section{SRM DIRECT TORQUE CONTROL BASED ON PHASE PLANE VARIABLE STRUCTURE CONTROL}

1.Torque characteristic analysis of SRM

From SRM circuit equation [9-10]:

$$
\psi_{k}=\int\left(V_{k}-R_{k} i_{k}\right) d t
$$

Therefore, the SRM flux linkage is a function of the voltage, current and winding resistance applied at both ends of the winding. Since the winding resistance $\mathrm{R}_{\mathrm{k}}$ is very small and can be ignored, equation (1) can be simplified as:

$$
\psi_{k}=\int V_{k} d t
$$

If the voltage time interval on the loading winding is $\Delta t$, the formula (3) is obtained:

$$
\Delta \vec{\psi}_{k}=\vec{V}_{k} \Delta t
$$

Equation (3) shows that the variation $\Delta \vec{\psi}_{k}$ of SRM stator flux vector is consistent with the direction of voltage vector $\vec{V}_{k}$ loaded on both sides of the stator and is directly proportional to the time added by the voltage vector.

When the magnetic circuit is saturated, the SRM torque can be written as:

$$
T(\theta, i)=i \sqcap \frac{\partial \psi(\theta, i)}{\partial \theta}
$$

It can be seen from equation (4) that the instantaneous torque of SRM is directly proportional to winding current and $\frac{\partial \psi(\theta, i)}{\partial \theta}$.

Since each phase of SRM is powered by DC power supply, the direction of current will not 
Article History: Received: 28 October 2021 Revised: 05 December 2021 Accepted: 10 January 2022 Publication: 28 February 2022

change, so the symbol of $T(\theta, i)$ torque is consistent with $\frac{\partial \psi(\theta, i)}{\partial \theta}$, indicating that $\frac{\partial \psi(\theta, i)}{\partial \theta}$ plays a decisive role in the instantaneous torque of motor. Under the condition that the current direction does not change, the torque characteristics of SRM are discussed as follows:

(1) When $\frac{\partial \psi(\theta, i)}{\partial \theta}>0, T(\theta, i)>0$, the stator flux is ahead of position $\theta$, and the motor generates positive torque;

(2), when $\frac{\partial \psi(\theta, i)}{\partial \theta}<0, T(\theta, i)<0$, the stator flux is ahead of position $\theta$, and the motor generates positive torque.

Therefore, when the current is constant, if the stator flux is ahead of $\theta$, the output torque of the motor can be increased. On the contrary, if the stator flux lags behind $\theta$, the output torque of the motor can be reduced. Therefore, the increase and decrease of motor torque can be controlled by flux linkage. From equation (3), it can be seen that the flux vector is directly proportional to the voltage vector, so the control flux is transformed into the adjustment of the voltage vector.

The goal of SRM direct torque control is to keep the amplitude of stator flux vector constant, control the torque by adjusting the phase angle between flux and flux, and change the acceleration or deceleration of flux vector to increase or decrease the torque, so as to reduce the torque ripple of SRM.

Discretize equation (3) to obtain:

$$
\vec{\psi}_{k}-\vec{\psi}_{k-1}=\vec{V}_{k} T_{s}
$$

To sum up, the purpose of SRM direct torque control is to ensure the constant amplitude of flux vector, and the purpose of torque regulation can be achieved by adjusting voltage vector. Therefore, reasonable selection of voltage vector applied at both ends of winding is the core problem of direct torque control strategy.

2.SRM direct torque control based on phase plane variable structure control

The basic idea of variable structure control (VSC) is to design a special hyperplane for the control system in the state space. Under certain conditions, the "structure" of the system is forced to move up and down with small amplitude and high frequency along the specified state trajectory, and slide to the equilibrium point on this hyperplane. Finally, it is asymptotically stable at the equilibrium point or in a specific area near the equilibrium point, that is, sliding mode motion, which is often called sliding mode control (SMC), as shown in Figure 1. 
Article History: Received: 28 October 2021 Revised: 05 December 2021 Accepted: 10 January 2022 Publication: 28

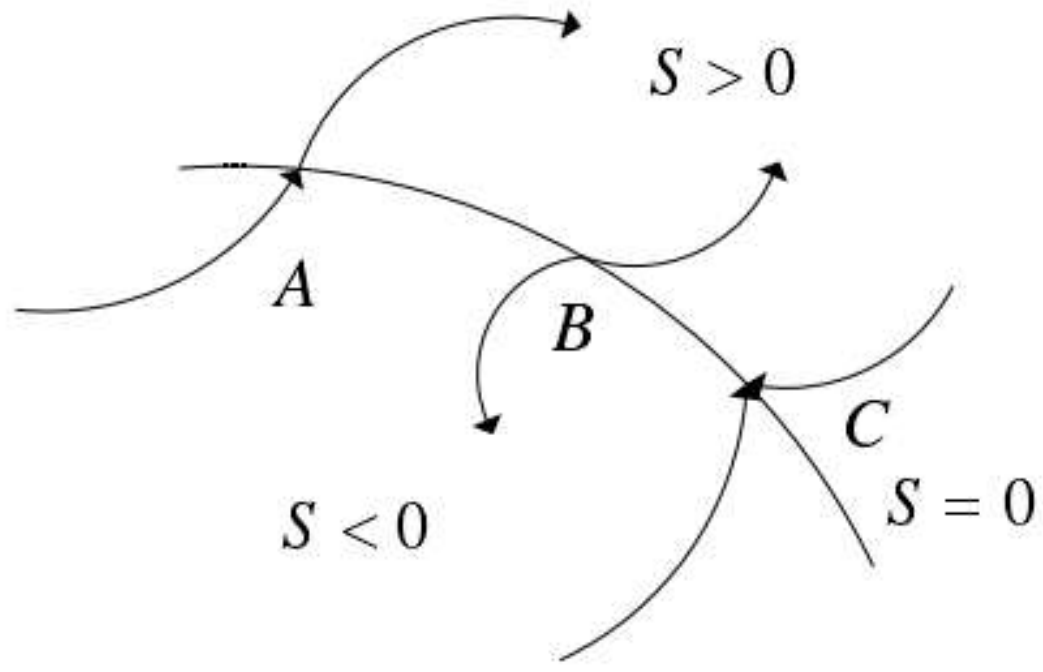

Fig 1: Schematic diagram of sliding mode control switching surface

Sliding mode control has the advantages of fast response and strong robustness. It has been popularized and applied in engineering practice such as power system control, robot control, flight control, satellite position control and so on.

The common sliding mode variable structure control has the following design methods:

(1) Constant switching control

$$
u=u_{0} \operatorname{sgn}(s(x))(6)
$$

Where $\mathrm{u}_{0}$ is the constant control quantity.

(2) Function switching control

$$
u=u_{e q}+u_{0} \operatorname{sgn}(s(x))
$$

(3) Proportional switching control

$$
\begin{gathered}
u=\sum_{i=1}^{k} \psi_{i} x_{i} \quad k<n \\
\psi_{i}= \begin{cases}\alpha_{i} & x_{i} s<0 \\
\beta_{i} & x_{i} s>0\end{cases}
\end{gathered}
$$

\section{SRM CONTROL BASED ON TORQUE CURRENT HYBRID MODEL}

1.Torque current model

The torque distribution control strategy finally comes down to the control of the current 
Article History: Received: 28 October 2021 Revised: 05 December 2021 Accepted: 10 January 2022 Publication: 28 February 2022

passing through the winding. Therefore, after the desired torque is distributed to each control phase through the torque distribution function, the torque signal needs to be converted into the winding current signal. The function of torque current model is to obtain the corresponding control current law by using the torque angle relationship of SRM. When the inductance change rate is positive, the torque has the following relationship with rotor position and phase current.

$$
T_{e}=\frac{1}{2} \frac{d L}{d \theta} i^{2}
$$

Equation (11) is obtained:

$$
i^{2}=\frac{2 T_{e}}{d L / d \theta}
$$

Since the current direction of SRM winding remains unchanged, it can be obtained that:

$$
i=\sqrt{\frac{2 T_{e}}{d L / d \theta}}
$$

Therefore, in the torque distribution control strategy, the torque current model is:

$$
i_{\text {ref }}=\sqrt{\frac{2 T_{k}(\theta)}{d L / d \theta}}
$$

The current controller adopts hysteresis control to track the reference current. The algorithm is simple and easy to implement. Under the SRM linear model, it is easy to obtain the torque current linear model, but this approximate treatment method can only reduce the torque ripple of SRM to a limited extent.

2.SRM control based on torque current hybrid model

The SRM linear model is convenient to analyze the torque characteristics of SRM, but it ignores the strong nonlinearity of SRM magnetic circuit and the saturation of local magnetic circuit. Therefore, the torque current model obtained by SRM linear model is only an approximate model, and its corresponding current law can only suppress the torque ripple of SRM to a certain extent.

Considering that the neural network has strong nonlinear fitting ability, a torque current hybrid model is established, in which RBFNN is connected in series after the torque current linear model. Through the nonlinear mapping ability of RBFNN, the reference current waveform is adjusted online to further reduce the torque ripple. The improved SRM torque distribution control system frame. In the system, three neural networks are introduced in series with the corresponding torque current linear model of SRM to obtain the best reference current signal of each corresponding phase under low torque ripple of three-phase SRM.

Under different speeds and loads, the corresponding constant torque of SRM is unknown, 
Article History: Received: 28 October 2021 Revised: 05 December 2021 Accepted: 10 January 2022 Publication: 28 February 2022

but the parameter adjustment of RBFNN in torque current hybrid model is based on torque ripple. How to establish the learning goal of RBFNN is a key problem in establishing torque current hybrid model.

When the speed and load fluctuate, the torque of SRM changes greatly, so it is impossible to obtain a stable tracking target. Therefore, in order to minimize the torque ripple in the whole process of SRM operation, the dynamic average value of SRM is extracted as the tracking target of RBF neural network. The deviation between SRM dynamic average torque and instantaneous torque is taken as the goal of RBFNN learning. When calculating the dynamic average torque, set the fixed time window $t$ as the period for calculating the SRM average torque, and obtain the arithmetic average value of the SRM output torque in the time window $t$ according to equation (14). The time window is shifted during the operation of SRM, so that the average value of torque is dynamically updated in real time, so as to realize the online learning of RBFNN.

$$
T_{a v}=\frac{1}{T} \int_{0}^{T} T(t) d t
$$

Where $\mathrm{t}$ is the calculation period and $\mathrm{T}(\mathrm{s})$ is the instantaneous torque output by SRM.

The rbfnn1 learning adopts the error feedback learning method, and the expression of its performance index function $\mathrm{E}(\mathrm{k})$ is as follows:

$$
E(k)=\frac{1}{2} e^{2}(k)=\frac{1}{2}(r(k)-y(k))^{2}
$$

Where, the torque reference signal $\mathrm{r}(\mathrm{k})$ is the dynamic average torque output by the motor, and $\mathrm{y}(\mathrm{k})$ is the instantaneous torque output by the motor.

\section{EXPERIMENTAL VERIFICATION OF SRM TORQUE DISTRIBUTION CONTROL}

In SRM control, the on-off of switching elements is realized according to the predetermined commutation logic. The commutation of SRM is closely related to the position signal. Therefore, accurate detection of position signal is the primary goal of SRM control. In the experimental platform, the position detection is realized by photoelectric sensor. In position detection, the photoelectric sensor is fixed on the machine body, and the rotary table is installed on the motor shaft to rotate with the motor. Each phase of the motor rotor corresponds to a photoelectric sensor, and the two ends of the photoelectric sensor slot are infrared light-emitting diode and infrared photoelectric triode respectively. When there is no shelter in the slot of the photoelectric sensor, the photoelectric triode can receive the light signal and conduct saturation; When the rotary table teeth turn to the center of the groove, the rotary table teeth block the transmission of light and cut off the photoelectric triode. After the conversion circuit, the photoelectric signal can be converted into a rotor position signal. System power circuit is shown 


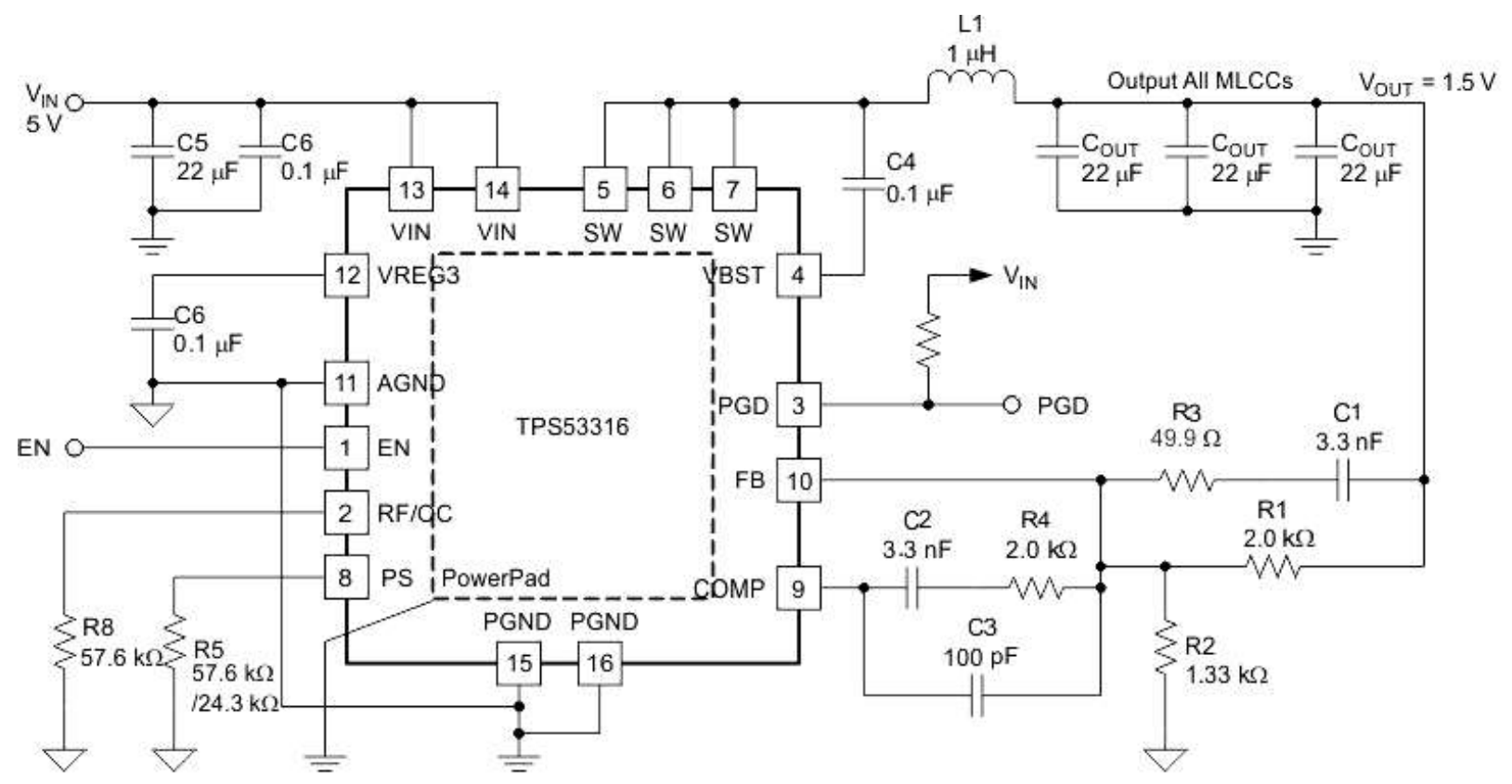

Fig 2: System power circuit

When SRM is started, due to the randomness of position, the cap module of DSP can not capture the falling edge of position signal and obtain rotor signal. Therefore, software is used to simulate the position detection signal in the SRM startup phase. The specific method is: when starting the motor, assuming that the A-phase position signal HA is captured, turn on the cphase according to the commutation logic. At the same time, T2 timing interrupt is enabled. If the rotor position signal is not captured after 30 consecutive timing interrupts, it is assumed that the current position signal is HC. Continue the above steps until the motor rotor rotation triggers the position sensor to generate a position signal, and the motor commutation is realized according to the position signal.

The phase current is usually used as the control quantity of SRM. Therefore, in the process of SRM control, the amplitude of current needs to be monitored in real time. Therefore, the phase current needs to be measured in the experiment. Measuring phase current requires ADC conversion unit of DSP. The ADC analog input range of DSP is $0 \sim 3 \mathrm{~V}$, so the maximum input voltage of sampling port is $3 \mathrm{~V}$. For analog signals with input signal greater than $3 \mathrm{~V}$, the voltage needs to be converted to within $3 \mathrm{~V}$ through conditioning circuit. In the experiment, the current sensor's measuring range is $0 \mathrm{~A} \sim 10 \mathrm{~A}$, and its input and output characteristics are:

$$
y=0.0625 x+2.5(16)
$$


Article History: Received: 28 October 2021 Revised: 05 December 2021 Accepted: 10 January 2022 Publication: 28 February 2022

Where $\mathrm{y}$ is the output voltage of the sensor and $\mathrm{x}$ is the sampling current value.

In order to verify the suppression effect of the proposed torque current hybrid model on SRM torque ripple, the simulation is carried out in Matlab / Simulink environment. According to the above motor model, two groups of simulation are designed. The first group adopts the traditional torque distribution control strategy, and the second group adopts the control strategy proposed in this paper. In the simulation control, the on angle, off angle and commutation angle in the torque distribution function are fixed. Referring to the SRM torque distribution method, the SRM control simulation diagram based on the torque current linear model shown in Figure 3 is designed in the environment of MATLAB / Simulink.

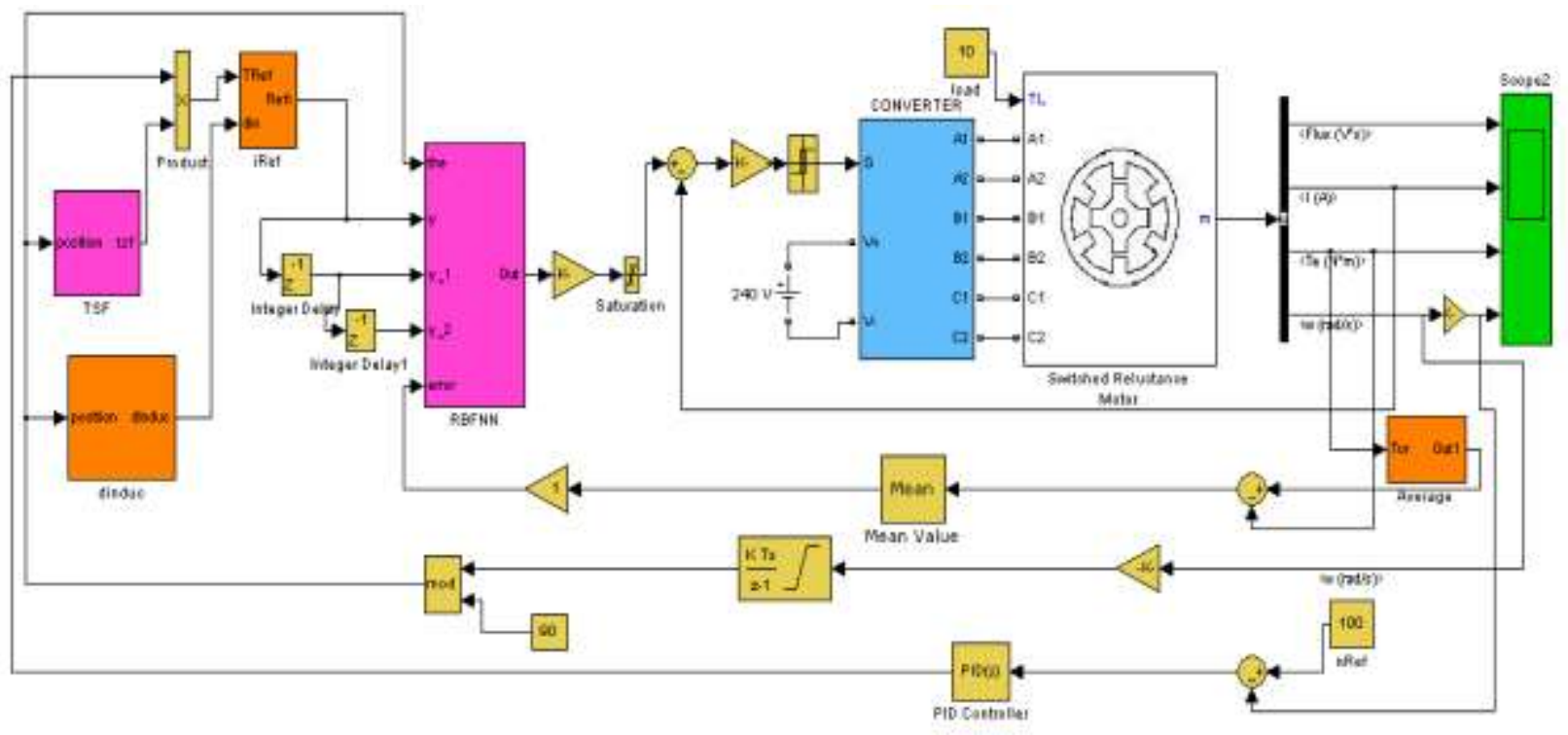

Fig 3: SRM control simulation diagram

Figure 4 shows the error waveform of dynamic average torque and instantaneous output torque when the SRM speed is 100r / min. the dotted line (gray) is the torque error waveform based on the torque current linear model, and the solid line (black) is the torque error waveform based on the torque current hybrid model. It can be seen from the figure that the torque error of the proposed SRM improved control system is smaller, and the torque variation is reduced from $\pm 0.4 \mathrm{~nm}$ to $\pm 0.18 \mathrm{~nm}$. 
Article History: Received: 28 October 2021 Revised: 05 December 2021 Accepted: 10 January 2022 Publication: 28 February 2022

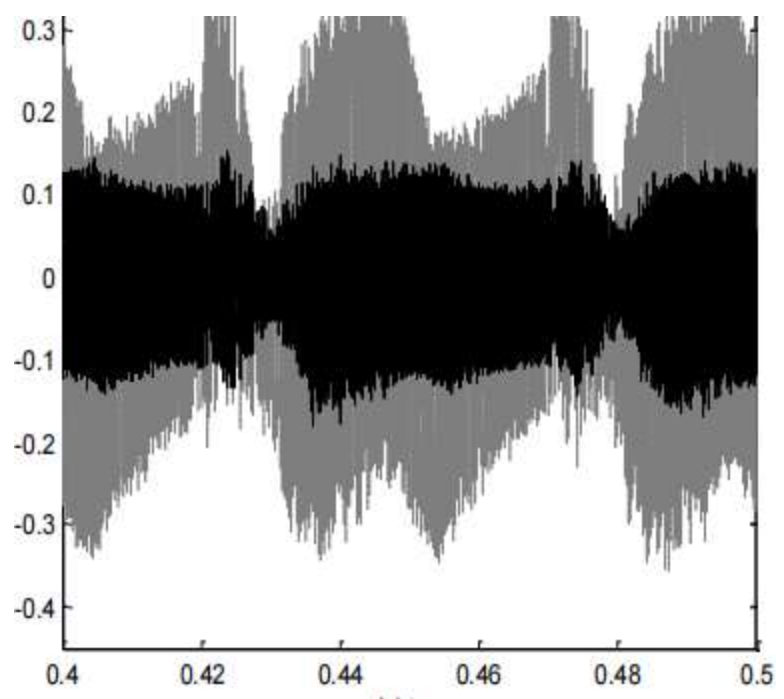

Fig 4: Simulation test results

The range and driving safety of pure electric vehicles are closely related to the performance of the driving system. As the core of the drive system, the selection of drive motor is particularly important. SRM has the advantages of low starting current, no hidden danger of short circuit in power circuit, low manufacturing cost and wide speed regulation range. It is an important choice to develop a new generation of pure electric vehicle. The special doubly salient structure and switching power supply mode of SRM lead to the nonlinearity of its magnetic circuit. This strong nonlinearity makes its output torque a strong nonlinear function of rotor position and current. Therefore, when the conventional control method is used to control SRM, it will produce large torque ripple, which limits its application in pure electric vehicles with high requirements for safety performance and comfort.

In the SRM torque distribution control strategy, the torque current linear model is used to map the torque current relationship. Because the torque of SRM is a nonlinear function of current and rotor position, the torque current model can not accurately reflect the mapping relationship between SRM torque and current. Therefore, a torque current hybrid model control strategy based on RBF neural network is proposed. Based on the deviation between the dynamic average value of SRM output torque and instantaneous torque, the online learning of RBF neural network is realized, and the torque current hybrid model is constructed. The RBF neural network connected in series with the torque current linear model realizes the nonlinear mapping from the torque current linear model of SRM to the torque current hybrid model corresponding to low torque ripple, and obtains the current variation law of SRM under low torque ripple operation. The simulation results show that the torque distribution control strategy based on torque current hybrid model further reduces the torque ripple of SRM.

\section{CONCLUSION}


Pure electric vehicle is the key direction of the development of new energy vehicles in China. It has the advantages of zero emission and energy saving. The development and promotion of pure electric vehicle technology is an important means to solve the shortage of fossil fuels such as oil in the future. SRM has the advantages of low starting current, no hidden danger of short circuit in power circuit, low manufacturing cost and wide speed regulation range. It is an important choice to develop a new generation of pure electric vehicle. Aiming at the torque ripple of SRM, this paper makes theoretical research on two different control strategies from direct torque control and torque distribution control: a phase plane variable structure control strategy is proposed to realize more effective control of SRM flux. The torque current hybrid model of SRM Based on RBF neural network is constructed, and the current variation law of SRM under low torque ripple operation is obtained.

\section{ACKNOWLEDGEMENTS}

This research was supported by Science and Technology Planning Project of Henan Province, China (Grant No. 212400410223)

\section{REFERENCES}

[1] Liu Xiangyang, Wang Dan. Design and Research of Electrical Automation Control System Based on Intelligent Technology. Electronic Design Engineering, 2019, V.27; No.414 (16): 7275

[2] Wen Wenhao. Research on Electrical Automation Control System Based on Artificial Intelligence. Information and Computer (theoretical Edition), 2020, V.32; No.446 (04): 152153

[3] Liu Xiaoyan. Application of Artificial Intelligence Technology in Electrical Automation Control System. Rural Economy and Technology, 2019, 030 (022): 254-255

[4] Guo Yanan, Wang Yanfei. Application of Artificial Intelligence Technology in Electrical Automation Control. Architectural Engineering Technology and Design, 2018, 16: 4184

[5] Huang Qiang. Application of Artificial Intelligence Technology in Electrical Automation Control. Consumer Guide, 2019, 10: 120

[6] Yin Zhangsheng. Research on Electrical Automatic Control Technology Under Artificial Intelligence Technology. Development Orientation of Building Materials, 2019, 017 (009): 363

[7] Wang Li. Application of Artificial Intelligence in Electrical Control System. Full Text Edition: Engineering Technology, 2016 (6): 175-175

[8] Jiang Fkuan, Zhou Rui. Discussion on the Application of Artificial Intelligence Technology in Electrical Automation Control. Dual Use Technologies and Products, 2018, 6: 96

[9] Yao Linhu. Application of Artificial Intelligence in Electrical Automation Control. Intelligent City Application, 2020, 3 (8): 91 
Article History: Received: 28 October 2021 Revised: 05 December 2021 Accepted: 10 January 2022 Publication: 28 February 2022

[10] Wang Huiqian. Application and Development of Artificial Intelligence Technology in Electrical Control System. Journal of College of Electronic Engineering, 2019, 008 (010): P.137-137 